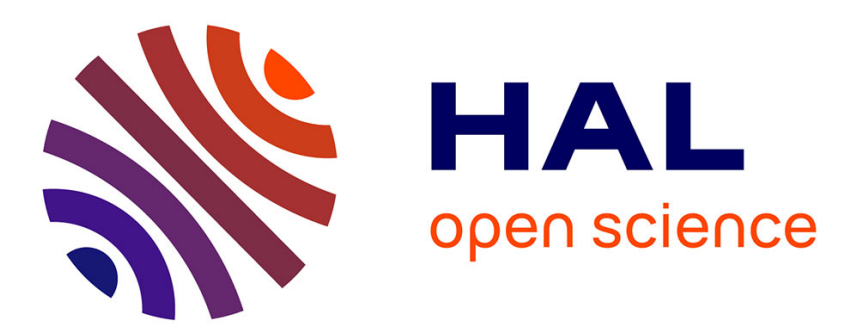

\title{
BEM-based models to simulate the resin flow at macroscale and microscale in LCM processes
}

Renaud Gantois, Arthur Cantarel, Benoit Cosson, Gilles Dusserre, Jean-Noël Felices, Fabrice Schmidt

\section{- To cite this version:}

Renaud Gantois, Arthur Cantarel, Benoit Cosson, Gilles Dusserre, Jean-Noël Felices, et al.. BEMbased models to simulate the resin flow at macroscale and microscale in LCM processes. Polymer Composites, 2013, 34 (8), pp.1235-1244. 10.1002/pc.22531 . hal-01687314

\section{HAL Id: hal-01687314 https://hal.science/hal-01687314}

Submitted on 8 Nov 2018

HAL is a multi-disciplinary open access archive for the deposit and dissemination of scientific research documents, whether they are published or not. The documents may come from teaching and research institutions in France or abroad, or from public or private research centers.
L'archive ouverte pluridisciplinaire HAL, est destinée au dépôt et à la diffusion de documents scientifiques de niveau recherche, publiés ou non, émanant des établissements d'enseignement et de recherche français ou étrangers, des laboratoires publics ou privés. 


\title{
BEM-Based Models to Simulate the Resin Flow at Macroscale and Microscale in LCM Processes
}

\author{
Renaud Gantois, ${ }^{1}$ Arthur Cantarel, ${ }^{2}$ Benoit Cosson, ${ }^{3,4}$ Gilles Dusserre, ${ }^{1}$ Jean-Noël Felices, ${ }^{2}$ \\ Fabrice Schmidt ${ }^{1}$ \\ ${ }^{1}$ Université de Toulouse, Mines Albi, INSA, UPS, ISAE , ICA (Institut Clément Ader), Campus Jarlard, \\ F-81013 Albi, France \\ ${ }^{2}$ Université de Toulouse, UPS, Mines Albi, INSA, ISAE , ICA (Institut Clément Ader), 1 rue Lautréamont, F- \\ 65016 Tarbes, France \\ ${ }^{3}$ UNIV Lille Nord de France, F-59000 Lille, France \\ ${ }^{4}$ Mines Douai, MPE-TPCIM, F-59508 Douai, France
}

In industry, resin flow prediction is a real challenge, because filling defects, such as dry spots and porosities, can dramatically degrade the mechanical properties of the shaped part. The present contribution investigates the use of the boundary element method for the numerical simulation of resin flow in liquid composite molding processes. Due to the dual-scale structure of the reinforcement, there are two different approaches in the literature to simulate the flow: the macroscale, well suited for the mold design, and the microscale, usually used for the local analysis of the reinforcement impregnation. In this work, both scales are under consideration. The article presents the numerical methods used to solve Darcy's law and Stokes equations with boundary integral formulations. Typical applications are given to illustrate both methods, and numerical validations are performed using comparisons with experimental and analytical data. The results show that the methods provide a high accuracy and a low CPU time.

\section{INTRODUCTION}

Liquid composite molding (LCM) refers to composite manufacturing processes involving a liquid resin flow through a dry fibrous reinforcement in a closed mold. It has been widely used in industry for the last decades to produce high quality and complex-shaped parts. Typical fields of applications can be found in automotive,

Correspondence to: Fabrice Schmidt; e-mail: fabrice.schmidt@mines-albi.fr aeronautics, spatial, marine, defense, or sporting goods industry. Resin transfer molding (RTM) is one of the most popular process among the large numbers of technologies currently in use, such as vacuum assisted RTM and liquid resin infusion. The present contribution focuses on the mold filling step of LCM process. In this step, the resin is forced into the mold to impregnate the fibrous reinforcement.

It has been proven that the quality of the impregnation determines the mechanical properties of the final composite material. In particular, a partial filling leaving dry spots and/or porosities within the reinforcement may dramatically decrease the mechanical performances of the part. This is why resin flow prediction has become a vital concern in composites manufacturing industry. To ensure a complete filling of the whole part, a special care is usually taken to place the injection ports and the vents in the mold. Cost of tools together with the time spent on developing the best filling strategy have led engineers to use numerical methods instead of full trial-and-error methods.

Numerical modeling addressing each step of the process, such as preform processing, mold filling and resin cure, has been extensively studied for the last 3 decades. In most cases, structural composites materials are manufactured using dual-scale porous reinforcements (typically carbon fabrics). The reinforcement architecture is usually made of a periodical organization of tows enclosing several thousands of fibers. Consequently, two different scales can be used to simulate the resin flow within the reinforcement: macroscale and microscale. The microscale focuses on the flow between the fibers inside the tow. Due to the large numbers of fibers, simulations are usually performed using representative elementary cells 
(REC). The size of these cells typically ranges from a few micrometers to a few millimeters, depending on material complexity and computational resources. Typical applications can be found in the field of numerical permeability computation using the Stokes equations [1,2]. On the contrary, macroscale is mainly used in the simulation of the whole composite part filling. Permeability is then utilized in the program as material input data, and the simulation is generally conducted using Darcy's law [3].

Most of the time, mold filling simulations are achieved in commercial codes using a control volume/finite element method (CV/FEM) [4]. This method is based on filling fractions, ranging from 0 (no resin) to 1 (completely filled with resin). Fractional values indicate that the resin/ air interfaces occurs in the cell. Several methods have been developed to improve the location of the interface. Among them, some techniques are based on reconstruction schemes, such as simple line interface calculation [5] or piecewise linear interface calculation [6], while others utilize some adaptive mesh refinements $[7,8]$ or Level Set Methods [9,10]. The Level Set Methods are advanced numerical techniques developed to capture complexshaped interfaces occurring in two and three dimensions. They are widely used in large range of numerical problems including propagating interfaces, for example, in fluid mechanics, crystal growth, combustion, and medical imaging.

This works investigates the use of boundary element method (BEM) to simulate the flows occurring at macroscale and microscale in the reinforcement. One important feature of the BEM is that it is only required to mesh the boundary to perform the computation, while a full domain mesh is needed in FEM methods. Therefore, BEM is well suited for large and complex-shaped domains. It is also well suited to combine with Level Set Methods, as they use the signed-distance to the interface to follow the front motion. The article presents the governing equations of the resin flow at both scales, and the main features of the numerical models. Finally, some typical applications illustrating the methods are given. Numerical results are also compared with experimental and analytical data.

\section{GOVERNING EQUATIONS}

In this work, it is assumed that the resin is Newtonian, and that the flow occurs in isothermal conditions. In particular, the filling time is assumed to be shorter than the time required to reticulate the resin, and viscosity is assumed to be constant and homogeneous. It is usually the case in injection and infusion processes since the resin has to be maintained at a low viscosity during the filling stage. It is also assumed that the fibers in the reinforcement are fixed and rigid during the resin motion. Besides, it is assumed that the permeability tensor is constant in time and space, although some fiber displacements may change the local properties of the reinforcement.

\section{Macroscopic Scale Flow}

It is generally admitted in the literature that the anisotropic form of Darcy's law is well suited to describe the macroscopic resin flow within the fibrous reinforcement [9,11]. In Darcy's law it is also implicitly assumed that the resin follows incompressibility equation. Therefore, the macroscale model is given as:

$$
\begin{cases}\vec{v}=-\frac{\overline{\bar{k}}}{\mu} \vec{\nabla} p & \text { in } \Omega \\ \vec{\nabla} \cdot \vec{v}=0 & \text { in } \Omega\end{cases}
$$

where $\Omega$ is the computational domain, $\vec{v}$ is the resin velocity, $p$ the pressure, $\mu$ the liquid resin viscosity, and $\overline{\bar{k}}$ the permeability tensor. It should be noticed that $E q$. 1a gives the superficial resin velocity, but the real velocity in the porous network can be computed using the fiber volume fraction of the dry reinforcement. For a twodimensional flow, the permeability tensor is given as follows:

$$
\overline{\bar{k}}=\left[\begin{array}{ll}
k_{11} & k_{12} \\
k_{12} & k_{22}
\end{array}\right]_{\left(\vec{e}_{1}, \vec{e}_{2}\right)}
$$

where $\left(\vec{e}_{1}, \vec{e}_{2}\right)$ denotes the unit vectors in twodimensional (2D) Cartesian coordinates. Two types of boundary conditions can be specified:

i. $p=\bar{p}$ on $\Gamma_{p}$ (Dirichlet condition)

ii. $q=\frac{\partial p}{\partial n}=\bar{q}$ on $\Gamma_{q}$ (Neumann condition)

where $\bar{p}$ (respectively, $\bar{q}$ ) is the prescribed value of pressure (respectively, normal derivative), and $\Gamma$ is the domain boundary, divided into parts at imposed pressure $\Gamma_{p}$ and parts at imposed normal derivative $\Gamma_{q}$ satisfying $\Gamma=\Gamma_{p} \cup \Gamma_{q}$.

\section{Microscopic Scale Flow}

The resin motion between the fibers (intra-tow domain) is governed by Stokes equations [1]:

$$
\begin{cases}\mu \Delta \vec{v}=\vec{\nabla} p & \text { in } \Omega \\ \vec{\nabla} \cdot \vec{v}=0 & \text { in } \Omega\end{cases}
$$

where $\Omega$ is the computational domain (resin), $\vec{v}$ the resin velocity, and $p$ the pressure. Two types of boundary conditions can be specified:

$$
\begin{aligned}
& \text { i. } v_{k}=\bar{v}_{k} \text { on } \Gamma_{v_{k}} \text { (imposed velocity) } \\
& \text { ii. } T_{k}=\bar{T}_{k} \text { on } \Gamma_{T_{k}} \text { (imposed stress) }
\end{aligned}
$$

where $v_{k}$ and $T_{k}$ are the prescribed $k$-component of velocity and stress vector on the boundary. The Newtonian resin behavior is given as follows: 


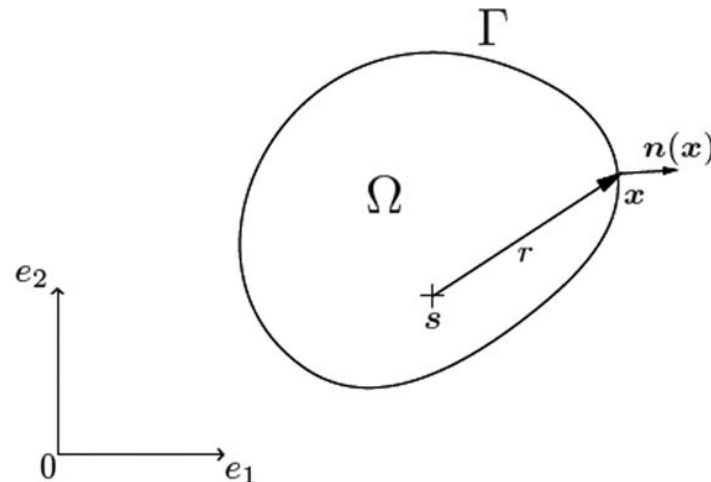

FIG. 1. Computational domain representation and geometrical notations in BEM.

$$
\sigma_{i j}=-p \delta_{i j}+2 \mu \dot{\gamma}_{i j}
$$

where $\sigma_{i j}$ is the stress tensor and $\dot{\gamma}_{i j}$ the strain rate tensor, given as follows:

$$
\dot{\gamma}_{i j}=\frac{1}{2}\left(\frac{\partial v_{i}}{\partial x_{j}}+\frac{\partial v_{j}}{\partial x_{i}}\right)
$$

where $x_{j}$ is the component $j$ (indicial notation). It should be mentioned that stress vector at boundary is given as $T_{i}=\sigma_{i j} n_{j}$, with $n_{j}$ being the component $j$ of outward normal.

\section{NUMERICAL METHOD}

This section covers the numerical technique used to solve the governing equations of the flows occurring at macroscale and microscale. The BEM [12-14] has been used in this work. The main feature of the BEM is the use of a boundary mesh instead of a full domain mesh. In this method, the solution at internal points is computed in a postprocessing step, once the boundary problem is solved. A Level Set method has been implemented in the macroscale model to advance the flow front inside the reinforcement during the mold filling stage. A Marching Triangles algorithm has also been utilized to generate a boundary mesh at each step time.

\section{BEM Resolution of Darcy's Law}

Boundary Integral Formulation. Combining Eqs. 1a and $1 \mathrm{~b}$ leads to the following equation:

$$
\left.\vec{\nabla} \cdot \quad-\frac{\overline{\bar{k}}}{\mu \varepsilon} \vec{\nabla} p\right)=0 \quad \text { in } \Omega
$$

where $\varepsilon$ is the porosity of the dry reinforcement, corresponding to the complementary value of the fiber volume fraction.

The boundary integral formulation is obtained using a Green's function, given as follows [12]:

$$
p^{*}(x, s)=\frac{1}{2 \pi \sqrt{\mid \overline{\bar{k} \mid}}} \ln \frac{1}{\hat{r}}
$$

where $p^{*}$ is the Green's function, computed as 2D fundamental solution of Eq. 6, $x$ and $s$ are the field point and the source point (Fig. 1), $|\overline{\bar{k}}|$ is the determinant of the permeability tensor, and $\hat{r}$ is a scalar function depending on domain geometry and permeability tensor:

$$
\hat{r}^{2}=\frac{1}{|\overline{\bar{k}}|}\left(k_{22} r_{1}^{2}-k_{12} r_{1} r_{2}+k_{11} r_{2}^{2}\right)
$$

where $r_{i}$ is the projection of $\vec{r}$ on outward normal $\vec{n}$ at boundary. Integrations by parts together with Green's theorem yield to Somigliana's equation:

$$
c(s) p(s)+\int_{\Gamma} q^{*}(x, s) p(x) d \Gamma(x)=\int_{\Gamma} p^{*}(x, s) q(x) d \Gamma(x)
$$

where $c(s)$ is the free term and $q^{*}$ the normal derivative of $p^{*}$. It should be mentioned that $E q .9$ is valid for any point source $s$ placed in the plane, in particular for internal points [15]. Therefore, pressure can be computed in the whole domain provided that both $p$ and $q$ are known along its boundary $\Gamma$. Next, the velocity can be computed using the derivative of Eq. 9.

Constant BEM. The numerical procedure consists in meshing the boundary using $N$ elements (Fig. 2). In the constant BEM, $p$ and $q$ are assumed to be constant and equal to the value at the mid-element node. The assembling process leads to a linear system given as $[H] \times \vec{P}=[G] \times \vec{Q}$, where $P_{i}$ (respectively, $Q_{i}$ ) is pressure (respectively, normal derivative of pressure) at node $i, H_{i j}$ and $G_{i j}$ are integration constants defined as follows:

$$
H_{i j}=\frac{1}{2} \delta_{i j}+\int_{\Gamma_{j}} q^{*}\left(x, x^{i}\right) d \Gamma_{j}(x)
$$

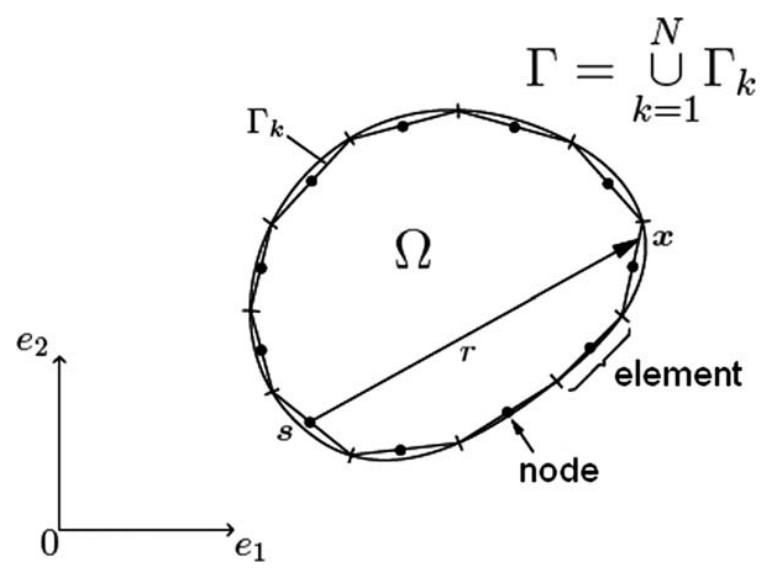

FIG. 2. Computational domain discretization using constant boundary elements. 
where $\delta_{i j}$ is the Kronecker symbol, and $\Gamma_{j}$ denotes the element $j$,

$$
G_{i j}=\int_{\Gamma_{j}} p^{*}\left(x, x^{i}\right) d \Gamma_{j}(x)
$$

Evaluation of coefficients $H_{i j}$ and $G_{i j}$ is achieved using a Gauss quadrature formula, except for the diagonal of matrix $H\left(H_{i i}=\frac{1}{2}\right)$. Boundary conditions are introduced into the system by assigning directly the prescribed nodal values, either $P_{i}=\bar{p}\left(x^{i}\right)$ or $Q_{i}=\bar{q}\left(x^{i}\right)$. Passing all unknowns on the left-hand side yields to the linear system ready to be solved, that is, $[A] \times \vec{X}=\vec{B}$.

\section{BEM Resolution of Stokes Equations}

Boundary Integral Formulation. The well known boundary integral formulation of elastostatics [12,13] can be used to solve the Stokes equations. However, displacement vector and strain tensor have to be replaced with velocity vector and strain rate tensor. Fundamental solutions are obtained by imposing a virtual Poisson coefficient of $\frac{1}{2}$ to satisfy the incompressibility condition. The virtual velocity is then given as follows:

$$
v_{l k}^{*}(x, s)=\frac{1}{4 \pi \mu}\left(\delta_{l k} \ln \frac{1}{r}+\frac{r_{l} r_{k}}{r^{2}}\right)
$$

and the virtual stress is given as:

$$
t_{l k}^{*}(x, s)=\frac{\left(r_{l} r_{k}\right)}{\pi r^{4}} \vec{r} \cdot \vec{n}
$$

Constant BEM. The numerical method used to assembly and solve the linear system is quite similar to the procedure described above. However, as fundamental solutions differ from the previous section, the linear system is now organized into the following matrix form:

$$
\begin{gathered}
{\left[\begin{array}{cccc}
{\left[H_{1,1}\right]} & {\left[H_{1,2}\right]} & \ldots & {\left[H_{1, N}\right]} \\
{\left[H_{2,1}\right]} & {\left[H_{2,2}\right]} & \ldots & {\left[H_{2, N}\right]} \\
\vdots & \vdots & \ddots & \vdots \\
{\left[H_{N, 1}\right]} & {\left[H_{N, 2}\right]} & \ldots & {\left[H_{N, N}\right]}
\end{array}\right]\left[\begin{array}{c}
{\left[V_{1}\right]} \\
{\left[V_{2}\right]} \\
\vdots \\
{\left[V_{N}\right]}
\end{array}\right]} \\
=\left[\begin{array}{cccc}
{\left[G_{1,1}\right]} & {\left[G_{1,2}\right]} & \ldots & {\left[G_{1, N}\right]} \\
{\left[G_{2,1}\right]} & {\left[G_{2,2}\right]} & \ldots & {\left[G_{2, N}\right]} \\
\vdots & \vdots & \ddots & \vdots \\
{\left[G_{N, 1}\right]} & {\left[G_{N, 2}\right]} & \ldots & {\left[G_{N, N}\right]}
\end{array}\right]\left[\begin{array}{c}
{\left[T_{1}\right]} \\
{\left[T_{2}\right]} \\
\vdots \\
{\left[T_{N}\right]}
\end{array}\right]
\end{gathered}
$$

where $\left[V_{i}\right]$ (respectively, $\left[T_{i}\right]$ ) is a $2 \times 1$ submatrix storing velocity vector (respectively, stress vector) at node $i$ and $\left[H_{i j}\right],\left[G_{i j}\right]$ are $2 \times 2$ submatrices given as:

$$
H_{i j l k}=\frac{1}{2} \delta_{i j} \delta_{l k}+\int_{\Gamma_{j}} t_{l k}^{*}\left(x, x^{i}\right) d \Gamma_{j}(x)
$$

where $(i, j)$ are the indexes of source point and the element over integration, and $(l, k)$ are indexes ranging from 1 to 2 .

$$
G_{i j l k}=\int_{\Gamma_{j}} v_{l k}^{*}\left(x, x^{i}\right) d \Gamma_{j}(x)
$$

All above submatrixes are evaluated numerically using a Gauss quadrature formula, except for node $i$ belonging to element $j$, for which the analytical solution is given as follows:

$$
H_{i i_{l k}}=\frac{1}{2} \delta_{l k}
$$

and

$$
G_{i i_{l k}}=\frac{L_{i}}{4 \pi \mu}\left[\left(1-\frac{L_{i}}{2}\right) \delta_{l k}+\frac{r_{l} r_{k}}{L_{i}^{2}}\right]
$$

where $L_{i}$ is the length of boundary element $i$. Boundary conditions are introduced the linear system by assigning the prescribed nodal values, that is, either $V_{i_{k}}=\bar{v}_{k}\left(x^{i}\right)$, or $T_{i_{k}}=\bar{t}_{k}\left(x^{i}\right)$.

\section{Resin Front Updating}

This section covers the case of a moving computational domain, that is, $\Omega=\Omega(t)$. In the implemented method, the boundary motion is computed using a Level Set method [16,17]. An important feature differing from $\mathrm{CV}$ methods is that the resin motion is fully governed by the boundary velocity field. Therefore, coupling Level Set with BEM is straightforward by meshing the advancing front at each time step. In the numerical model, the boundary mesh used in the BEM is defined as the intersection between the flow front and another grid of higher dimension, also called background grid. A Marching Triangles algorithm has been implemented to generate the boundary mesh $[18,19]$.

Signed Distance Function. In Level Set Methods, the boundary is embedded in a signed distance function, so that the zero Level Set corresponds to the interface [16]:

$$
\Gamma(t)=\left\{x \in I R^{2} / \phi(x, t)=0\right\}
$$

Distance is signed negative if the point is located in the impregnated region, or it is signed positive otherwise:

$$
\phi=\left\{\begin{array}{lc}
-d(x, \Gamma) & \text { if } x \text { inside } \Omega \\
+d(x, \Gamma) & \text { if } x \text { outside } \Omega
\end{array}\right.
$$

where $x$ is the node under consideration and $d(x, \Gamma)$ the Euclidean distance from that point to the front $\Gamma$. In this 


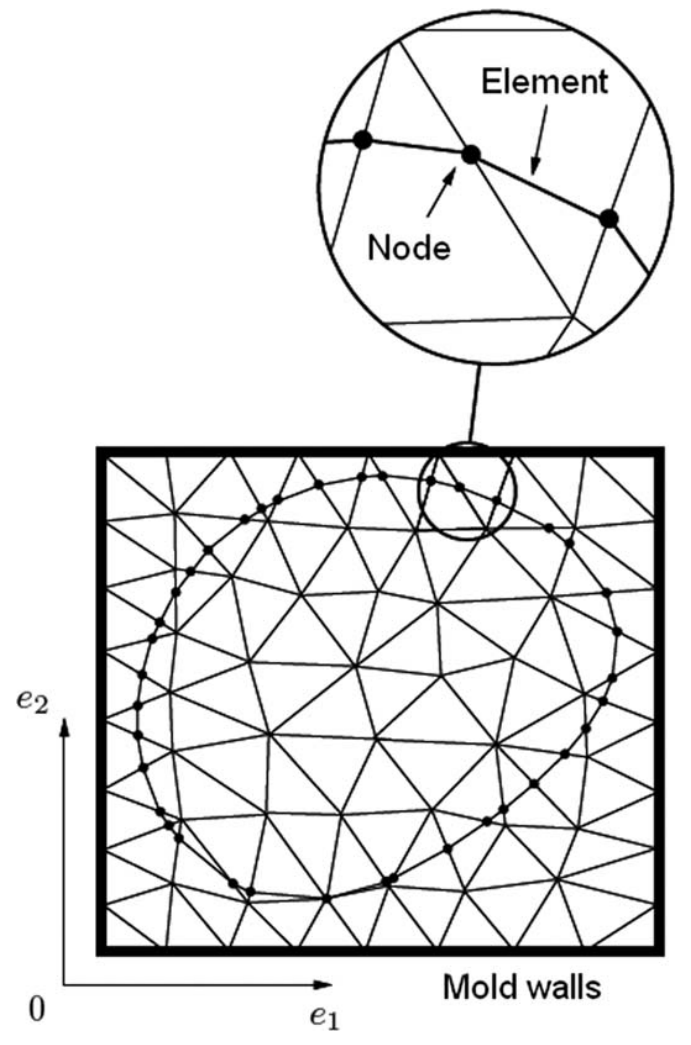

FIG. 3. Boundary elements mesh of the front using Marching Triangle algorithm.

work, the background grid is generated using a free triangulation with 3-nodes triangles (linear interpolation) (Fig. 3).

Level Set Formulation and BEM Coupling Strategy. The Level Set equations are used to govern the evolution of the signed distance function. To combine with BEM, the formulation involving a propagating interface with a velocity in its normal direction is used. It is given as follows:

$$
\left\{\begin{array}{l}
\frac{\partial \phi}{\partial t}+F|\nabla \phi|=0 \\
\phi(x, t=0)=\phi_{0}
\end{array}\right.
$$

where $F$ is the norm of the extended normal velocity, computed according to the nearest neighbor rule: for each background node, the velocity corresponding to the nearest computational boundary node is assigned (for further details see Ref. [17]).

Updating Scheme. At the beginning of the filling stage, $\phi_{0}$ is evaluated by computing the signed distance from the background nodes to the inlet gates. Next, the signeddistance function is updated using the following expression (Euler scheme):

$$
\phi(x, t+\Delta t)=\phi(x, t)-F(x, t)|\nabla \phi(x, t)| \Delta t(t)
$$

where $\Delta t$ is the time step, adjusted to match its upper limit according to the Courant-Friedrichs-Levy conditions. These conditions restrict the motion of the front to one grid cell per step time.

Reinitialization. Level Set field tends to lose its signeddistance property while being updating. Reinitialization step, usually performed by solving a Hamilton-Jacobi system, is required to maintain the property. In the present contribution, reinitialization is carried out by computing explicitly Euclidean distances from grid to boundary mesh. For sake of efficiency, the reinitialization should be restricted in a narrow-band around the front.

Boundary Remeshing Procedure. The boundary mesh is updated at each time step using the background grid through a Marching Triangles algorithm [18]. This algorithm has two advantages comparing with the standard Marching Squares: ambiguous intersecting cases vanish, and grid generation can be performed using some free triangulation methods implemented in most of commercial meshing softwares.

In this algorithm, each triangle is visited to determine how the front intersects the grid. Eight intersection cases can be encountered, but they reduce to only two topologies using symmetry and nodes permutations. The lookup table is defined by labeling each triangle vertices either outside or inside the fluid domain. In the implemented algorithm, once the intersecting case is identified, boundary nodes are placed by linearly interpolating the zero Level Set along the triangle edges (Fig. 3). Boundary elements are created by connecting two nodes using a straight line. As connections occur within a triangle, it is straightforward to generate the connectivity of the elements in the mesh. Normals to elements are oriented outward using the sign of the signed-distance function within the triangle.

It is important to notice that the above method generally leads to a large number of elements in the boundary mesh. However, it is easy to reduce significantly unnecessary elements (typically found near corners of triangles) using a perturbation procedure acting on the Level Set data [20].

Contact Implementation. The method described above directly handles topological changes appearing when fronts merge. However, it does not ensure that the resin remains inside the cavity. The nonpenetration condition is implemented using a fixed Level Set describing the walls. At each time step, the current Level Set is modified by intersecting mold with resin front using the following expression: 


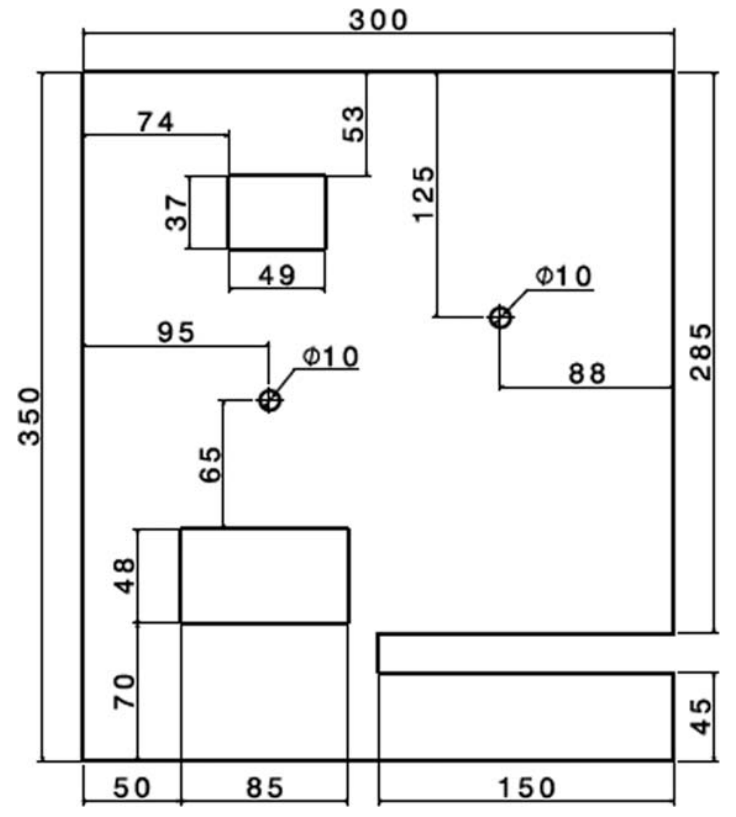

FIG. 4. Dimensions of the mold.

$$
\phi=\max \left(\phi, \phi_{m}\right)
$$

where $\phi_{m}$. mold is the signed-distance function to the mold walls.

\section{APPLICATIONS}

Applications illustrating the macroscale and microscale numerical models are presented in this section. Some experimental data and analytical solutions are used to assess and discuss the results.

\section{Knitted Fabric Impregnation}

To validate the numerical results, an experimental setup was developed to follow the infusion of an anisotropic knitted fabric. The fabric was multi-injected and fitted with internal obstacles acting like inserts to get closer from industrial concerns. Geometry and dimensions of the mold are given Fig. 4.

Experimental Procedure. The fabric used to perform the test is a glass fiber material knitted with a $1 \times 1$ rib pattern (manufactured by Textile Aéro Tarn). During the test, the resin was replaced with a model fluid used at room temperature in order to maintain isothermal conditions during the flow. For that purpose, canola oil was chosen. This oil has been used in literature by the past for its properties closed to typical thermoset resin

TABLE 1. Material data.

\begin{tabular}{lccc}
\hline$K_{1}\left(\mathrm{~m}^{2}\right)$ & $K_{2}\left(\mathrm{~m}^{2}\right)$ & $\mu(\mathrm{Pa} \mathrm{s})$ & $\varepsilon(-)$ \\
\hline $1.50 \mathrm{e}-9$ & $7.75 \mathrm{e}-10$ & 0.1 & 0.705 \\
\hline
\end{tabular}

TABLE 2. Processing parameters.

\begin{tabular}{lcc}
\hline $\begin{array}{l}\text { Inlet pressure: } \\
p_{0}(\mathrm{~Pa})\end{array}$ & $\begin{array}{c}\text { Outlet pressure: } \\
p_{\mathrm{f}}(\mathrm{Pa})\end{array}$ & $\begin{array}{c}\text { Inlet gate radius: } \\
r_{0}(\mathrm{~m})\end{array}$ \\
\hline $1.03 \mathrm{e} 5$ & 120 & $5 \mathrm{e}-3$ \\
\hline
\end{tabular}

(viscosity of $0.075 \mathrm{~Pa} \mathrm{~s}$ at $20^{\circ} \mathrm{C}$ and superficial tension of $33.4 \mathrm{mN} \mathrm{m}^{-1}$ ) and its perfect Newtonian behavior [21]. Material data and processing parameters used to conduct the test are summarized in Tables 1 and 2. It should be noted that permeability tensor was previously measured using a transient in-plane method: a complete description of the measurements is available in Ref. [21].

The experimental set-up consisted in infusion process equipment equipped with pressure sensors and a camera coupled device (CCD) camera placed in front of the reinforcement (Fig. 5). The knitted fabric (one layer) was put in place under a transparent flexible bag and sealed on a tooling plane surface using a vacuum sealant tape. It should be mentioned that no draining product was used in the set-up for the need of the test. The canola oil was stored at room temperature in an open container at atmospheric pressure. In the infusion set-up, the main flow was divided into two injection lines in order to obtain two separates ports for the canola oil entering the reinforcement. A constant vacuum was maintained inside the cavity in order to control the pressure during the flow. For that purpose, two vacuum lines were placed on the part and connected to the pump. The camera used to follow the flow front was a $1,350 \times 1,024 \mathrm{px}^{2} \mathrm{CCD}$ with typical magnification factor of images of $2.7 \mathrm{px} \mathrm{mm} \mathrm{mm}^{-1}$. The frame rate used to collect the data was four images per second.

Numerical Procedure. The background grid used to mesh the reinforcement includes 560 nodes and 4,624 elements and was generated with a free triangulation method using IDEAS (Fig. 6). For the sake of illustration, the Level Set corresponding to the signed distance to the mold is given together with the mesh.

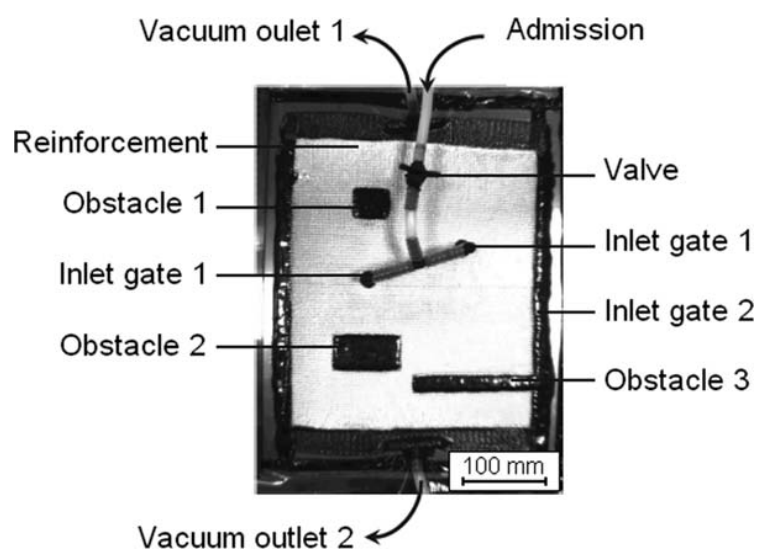

FIG. 5. Experimental setup. 


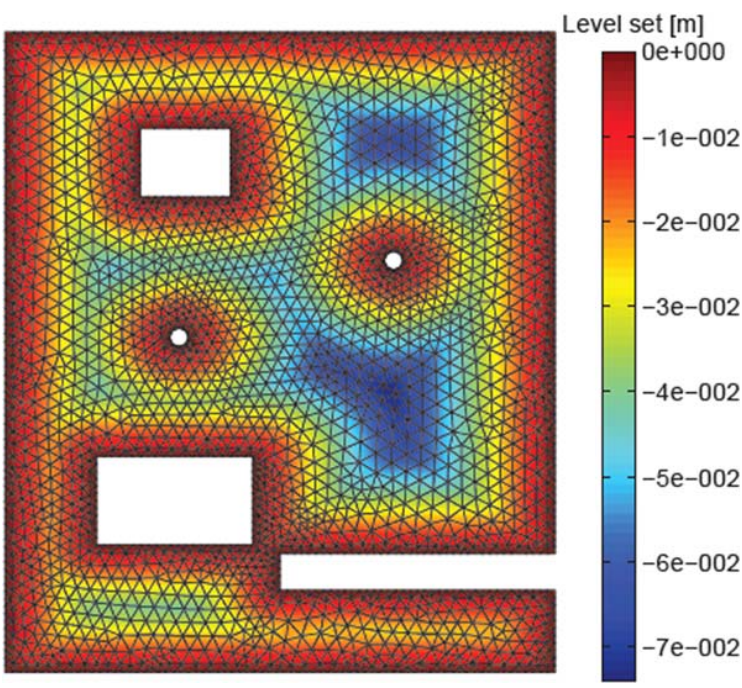

FIG. 6. Background grid and Level Set of the mold.

Boundary conditions have been assigned as follows:

i. $p=p_{0}$ at inlet and $p_{f}$ at outlet

ii. $q=0$ on mold walls and inserts (non penetration condition)

CPU time for the whole computation was $25 \mathrm{~s}$ using a P8400 Intel Core laptop (2.26 GHz, 1.9 GB of RAM). The resolution was fully conducted using MATLAB environment.

Results and Discussion. At the end of the test, some images have been utilized to make a comparison between experience and simulation at different times (Fig. 7). For that purpose, an experimental Level Set was computed to be compared with the numerical Level Set used in the program. For each image, the front location was extracted using an image analysis procedure developed using MATLAB environment. Even if it is not necessary to compute a full domain solution to obtain the numerical filling patterns, pressure at internal points has also been computed for the sake of illustration. This step has been achieved in a postprocessing routine based on $E q .15$.

The comparison between experimental and numerical Level Sets revealed a good accordance: numerical front patterns did not differ more than $20 \mathrm{~mm}$ from images. Besides, fronts merging as well as contact with internal obstacles were successfully predicted by the program. It should be mentioned that the numerical method provided a fair description of the front shapes owing to the use of Level Set method in the front capturing implementation.

The impregnated areas were also compared in order to check that mass variations were correctly computed. The impregnated pixels on each image were counted through an image analysis procedure. Results reveal a good agreement in the whole filling stage (Table 3). In particular, once the first seconds were elapsed, differences were less than $7 \%$, in spite of geometry approximations and edge effects along the mold walls. However, it should be noted that higher differences were reached during the first seconds of the flow. This may be explained by a high sensitivity to time synchronization and inlet ports geometry approximations.

\section{Computation of Transverse Permeability in REC}

The saturated flow perpendicular to the fibers has been computed using different REC. Next, the boundary velocity field was used to compute the transverse permeability using Darcy's law.

Quadratic Fiber Packing. The resin flow through unidirectional reinforcements has been studied by Gebart [22]. In his model, the author uses the thin channel flow assumptions to obtain a closed-form solution of Stokes equations in a quadratic fiber packing. It is also assumed that fibers are fixed and that sticking contact occurs between fibers and resin. The following expression is proposed for the transverse permeability:

$$
K_{\mathrm{t}}=\frac{16}{9 \pi \sqrt{2}}\left(\sqrt{\frac{\pi}{4 V_{\mathrm{f}}}}-1\right)^{\frac{5}{2}} R^{2}
$$

where $K_{\mathrm{t}}$ is the transverse permeability, $R$ the fiber radius, $V_{\mathrm{f}}$ the fiber volume fraction and $\frac{\pi}{4}$ the maximum fiber volume fraction reachable in a quadratic packing.

This solution has been used to assess the numerical model. A parametric study was achieved using a wide range of fiber volume fraction varying between 0.1 (poor fiber content) and 0.7 (high fiber content). Fiber radius has been maintained constant and equal to $3.5 \mu \mathrm{m}$ (typical radius of carbon fiber). The fiber volume fraction has been set by decreasing or increasing the gap between the fibers. For symmetry reasons, several patterns can be considered to carry out the calculations [2]. This study has been achieved using the smallest pattern, including a quarter of fiber.

The boundary conditions are given below. They have been assigned following Gebart's assumptions. In particular, a constant pressure flow has been imposed to drive the flow within the microstructure.

i. $\bar{v}_{k}=0$ at fiber/resin interfaces

ii. $\bar{T}_{k}=-\bar{p} n_{k}$ at REC inlet and outlet boundaries

iii. $\bar{T}_{1}=0$ and $\bar{v}_{2}=0$ at REC upper and lower boundaries

where $\bar{p}$ is a prescribed value of pressure, equal to $p_{\text {in }}$ at inlet and $p_{\text {out }}$ at outlet. It should be noted that conditions (i) stands for an impermeable and sticking contact whereas condition (iii) stands for a slipping contact 

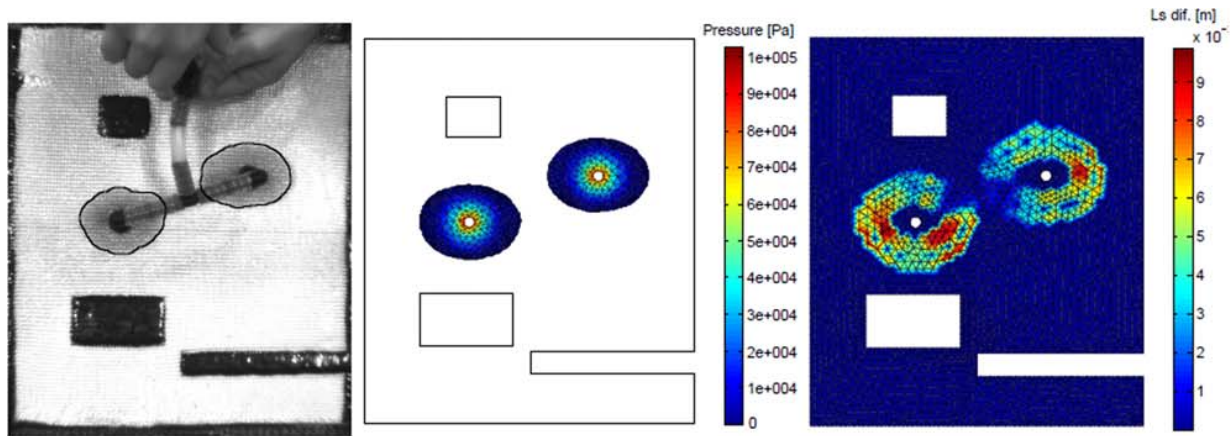

(a) Time :0.5 s
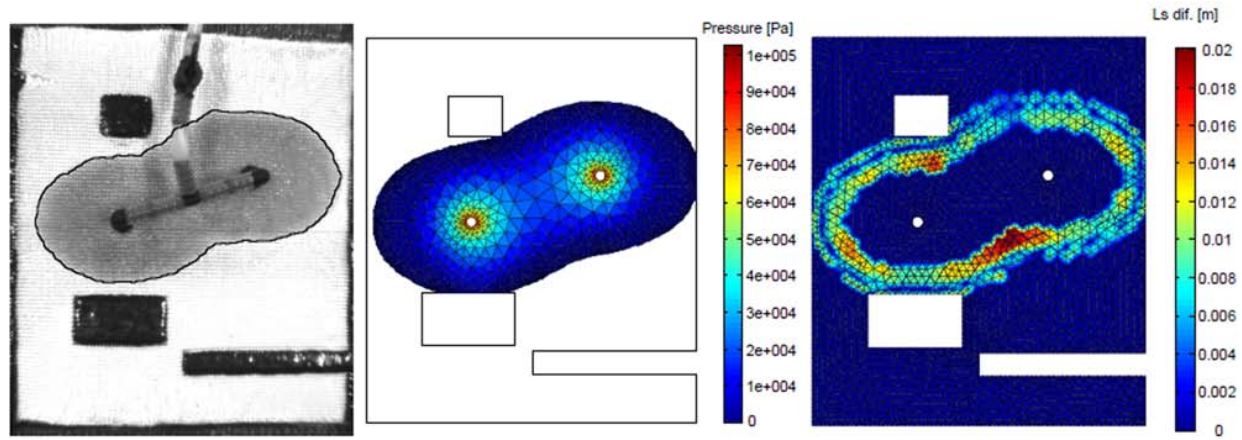

(b) Time : $3 \mathrm{~s}$
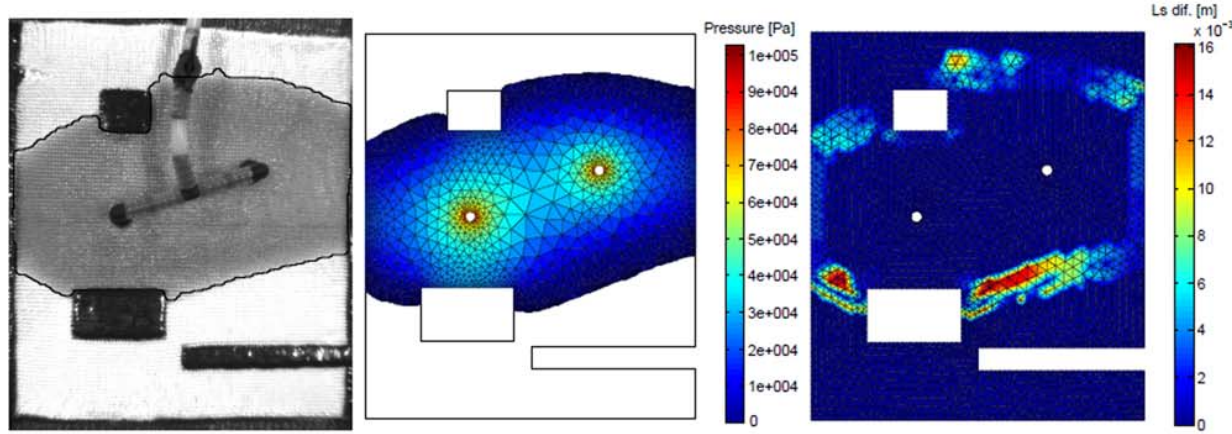

(c) Time : $5 \mathrm{~s}$
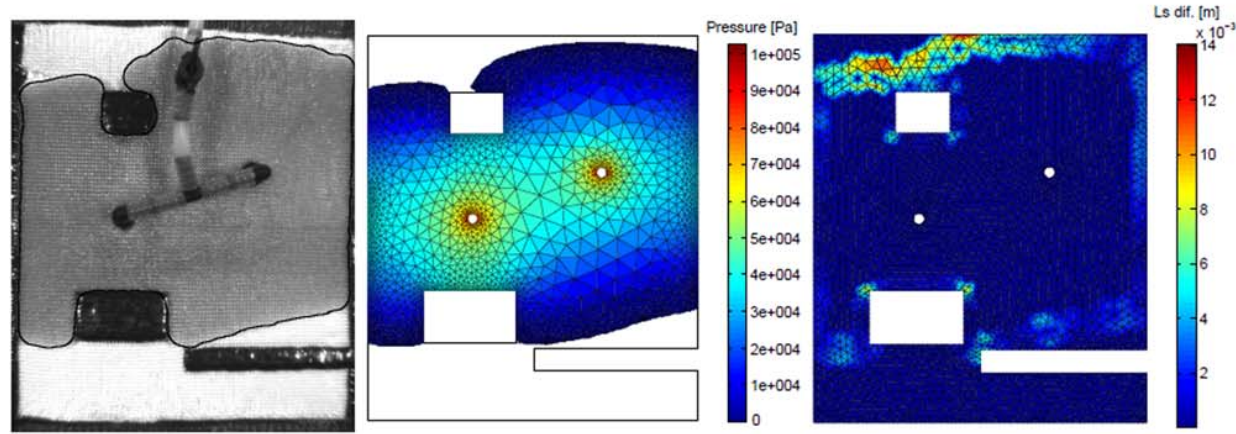

(d) Time: $8 \mathrm{~s}$

FIG. 7. Comparison between experimental and numerical filling patterns.

corresponding to symmetry conditions. Pressures and viscosity used in the simulation are given in Table 4 .

At the end of the calculation, the transverse permeability was computed using of the flow rate exiting the REC. Darcy's law yields to the following expression:

$$
K_{\mathrm{t}}=\frac{\mu\left(1-V_{\mathrm{f}}\right)}{p_{\text {in }}-p_{\text {out }}} \int_{\Gamma_{\text {out }}} \vec{v} \cdot \vec{n} d \Gamma
$$

where $\Gamma_{\text {out }}$ is the boundary at the exit of the REC under consideration. 
TABLE 3. Impregnated area: comparison between experimental and numerical data.

\begin{tabular}{lccc}
\hline Time $(\mathrm{s})$ & Exp. $\left(\mathrm{mm}^{2}\right)$ & BEM $\left(\mathrm{mm}^{2}\right)$ & Difference $(\%)$ \\
\hline 0.5 & $7.23 \mathrm{e} 3$ & $8.32 \mathrm{e} 3$ & 15.0 \\
1.5 & $1.48 \mathrm{e} 4$ & $1.90 \mathrm{e} 4$ & 28.7 \\
3.0 & $2.89 \mathrm{e} 4$ & $3.52 \mathrm{e} 4$ & 21.2 \\
4.0 & $4.07 \mathrm{e} 4$ & $4.35 \mathrm{e} 4$ & 6.9 \\
5.0 & $4.91 \mathrm{e} 4$ & $4.91 \mathrm{e} 4$ & 0.1 \\
6.0 & $5.55 \mathrm{e} 4$ & $5.52 \mathrm{e} 4$ & 0.5 \\
7.0 & $6.29 \mathrm{e} 4$ & $5.90 \mathrm{e} 4$ & 6.2 \\
8.0 & $6.57 \mathrm{e} 4$ & $6.96 \mathrm{e} 4$ & 6.0 \\
\hline
\end{tabular}

TABLE 4. Driving pressures and viscosity.

\begin{tabular}{lcc}
\hline$p_{\text {in }}(\mathrm{Pa})$ & $p_{\text {out }}(\mathrm{Pa})$ & $\mu(\mathrm{Pa} \mathrm{s})$ \\
\hline $2 \mathrm{e} 5$ & $1 \mathrm{e} 5$ & 0.1 \\
\hline
\end{tabular}

Results of the study are reported in Fig. 8. Dimensionless permeability has been computed for sake of generality. The parametric study revealed an excellent accordance between Gebart's model and numerical results in the whole range of fiber volume fractions. However, the better results have been obtained for high fiber volume fractions due to the thin flow channel assumption used in Gebart's approach.

Stochastic Fiber Distribution. In most cases, the fibers arrangement is disordered and heterogeneous. Several authors investigated numerically the effect of a stochastic distribution of fibers on the permeability [1,10]. The method developed by Chen and Papathanasiou [1] was used in this work to generate different microstructures using a random sampling. The process is divided in two main steps. In the first step, the microstructure is

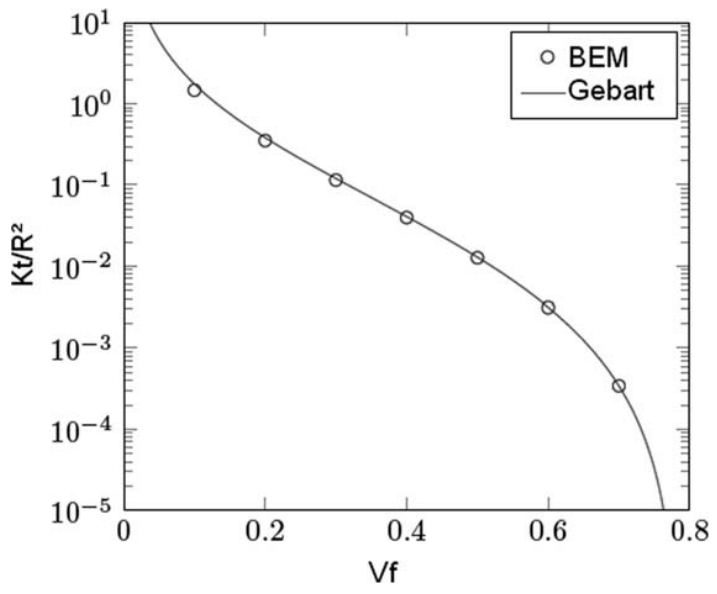

FIG. 8. Transverse permeability versus fiber volume fraction.

initialized in a regular quadratic packing of specified fiber volume fraction. Next, the fibers are randomly displaced through a Monte-Carlo method, until the microstructure reaches a disordered arrangement.

The simulation was performed on a square REC of $100 \mu \mathrm{m}$ side using a fiber volume content of 0.25 . In the numerical model, fibers sections were assumed to be circular of equal radius set to $3.5 \mu \mathrm{m}$ (carbon fiber). This REC was meshed using 988 boundary elements, with 12 elements for each fiber (Fig. 9). Boundary conditions are identical to the previous case of quadratic fiber packing (see above). Two simulations were conducted in the REC, one in each directions of the plane.

CPU time for whole computation was less than $1 \mathrm{~min}$ on a laptop using MATLAB (2.26 GHz, 1.9 GB of RAM). The boundary velocity field in each direction of the flow is given Fig. 9. The results reveal that the resin clearly skirts around the fibers and slows down close to them,

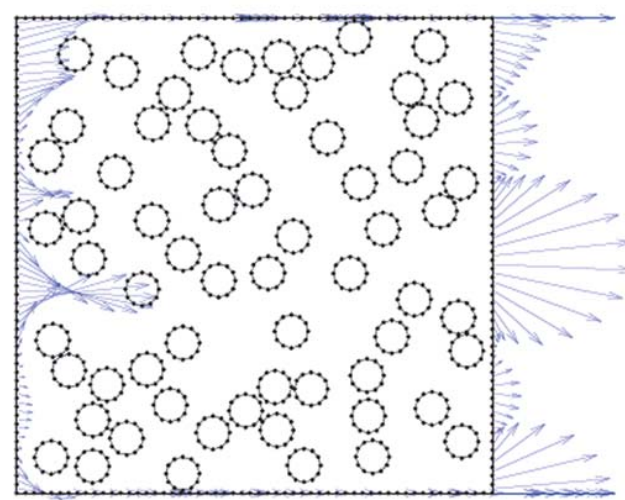

Flow in direction 2

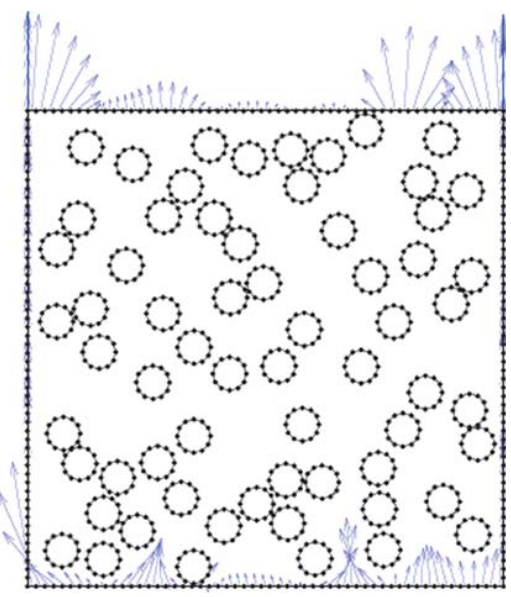

Flow in direction 3

FIG. 9. Boundary velocity in the REC. 
acting like rigid and sticking obstacles, and some preferential flows occur in regions of poor fiber content. The microstructure under consideration is slightly anisotropic (permeability ratio of 0.96 ), with numerical values of permeability of $1.83 \times 10^{-12} \mathrm{~m}^{2}$ in direction 2 and $1.90 \times$ $10^{-12} \mathrm{~m}^{2}$ in direction 3 . Comparing with a quadratic fiber packing $\left(2.57 \times 10^{-12} \mathrm{~m}^{2}, E q\right.$. (24)) these values are slightly lower, underlining the importance of considering a REC similar to the actual fiber arrangement.

\section{CONCLUSIONS}

The article investigates two BEM-based methods to simulate the macroflow and microflow occurring in the dual-scale structure of the reinforcement. The numerical models developed in this works are 2D and the flow is assumed to be isothermal.

At macroscale, the numerical model is based on a BEM/ Level Set coupling. An anisotropic knitted fabric has been impregnated with a Newtonian model fluid in order to validate the method. For that purpose, an experimental procedure has been developed to follow the front progression in an infusion setup. The results are in good accordance with the experimental data. In particular, the front shape is accurately predicted while the $\mathrm{CPU}$ time remains quite low.

In the microscale model, Stokes equations are solved using a BEM method based on elastostatics Green's function. Intra-tow saturated permeability is computed using different REC. The simulation of the flow within a quadratic pattern reveals a good accordance with Gebart's model. Current works investigates a stochastic distribution of fibers and elliptical shapes - dual scales flow involving both intra and inter tow should also be tested.

\section{ACKNOWLEDGMENTS}

This work was carried out in the framework of FUSCOMP project coordinated by DAHER-Socata. The authors are grateful to S. Leroux and L. Silva for their experimental and numerical contributions during this work. They also acknowledge N. Pirc, M. Bordival, F. Berthet, O. Pascal, and R. Beltrame for their implication and all helpful discussions.

\section{REFERENCES}

1. X. Chen and T. Papathanasiou, Compos. Sci. Technol., 67, 1286 (2007).
2. L. Silva, G. Puaux, M. Vincent, and P. Laure, Int. J. Mater. Forming, 3, 619 (2010).

3. H. Darcy, Les Fontaines Publiques de la Ville de Dijon (in French), V. Dalmont, Paris (1856).

4. C.W. Hirt and B.D. Nichols, J. Comput. Phys., 39, 201 (1981).

5. W.F. Noh and P. Woodward, in Proceedings of the Fifth International Conference on Numerical Methods in Fluid Dynamics, Twente University, Enschede (1976).

6. J. Li., Calcul d'interface affine par morceaux (Piecewise Linera Interface Calculation), Compte Rendus de l'Académie des Sciences, Ilb, Paris, France (1995).

7. E. Bechet, E. Ruiz, F. Trochu, and J.-C. Cuilliere, J. Reinforced Plast. Compos., 23, 17 (2004).

8. S. Soukane and F. Trochu, J. Reinforced Plast. Compos., 24, 1629 (2005).

9. S. Soukane and F. Trochu, Compos. Sci. Technol., 66, 1067 (2006).

10. F. Zhang, B. Cosson, S. Comas-Cardona, C. Binetruy, Compos. Sci. Technol., 71, 12 (2011).

11. P. Simacek and S.G. Advani, Compos. A, 37, 1970 (2006).

12. C.A. Brebbia and J. Dominguez, Boundary Elements: An Introductory Course, 2nd ed., McGraw-Hill Company: Computational Mechanics Publications, New York (1992).

13. F. Paris and J. Canas, Boundary Element Method: Fundamentals and Applications, Oxford University Press (1997).

14. F.M. Schmidt, P. Lafleur, F. Berthet, and P. Devos, Polym. Compos., 20, 725 (1999).

15. K.H. Yu, A.H. Kadarman, and H. Djojodihardjo, Eng. Anal. Boundary Elem., 34, 884, (2010).

16. J.A. Sethian, Level Set Methods and Fast Marching Methods Evolving Interfaces in Computational Geometry, Fluid Mechanics, Computer Vision, and Materials Science, 2nd ed., Cambridge University Press (1999).

17. J.A. Sethian and P. Smereka, Ann. Rev. Fluid Mech., 35, 341 (2003).

18. T.S. Newman and H. Yi, Comput. Graphics, 30, 854 (2006).

19. W.E. Lorensen and H.E. Cline, Comput. Graphics, 21, 163 (1987).

20. O. Seungtaik and B.K. Koo, Graphical Models, 69, 211 (2007).

21. G. Dusserre, E. Jourdain, and G. Bernhart. Polym. Compos., 32, 18 (2011).

22. B.R. Gebart, J. Compos. Mater., 26, 1100 (1992). 